\title{
On a New Cournot Duopoly Game
}

\author{
H. N. Agiza, ${ }^{1}$ A. A. Elsadany, ${ }^{2}$ and M. M. El-Dessoky ${ }^{1,3}$ \\ ${ }^{1}$ Department of Mathematics, Faculty of Science, University Mansoura, Mansoura 35516, Egypt \\ ${ }^{2}$ Department of Basic Science, Faculty of Computers and Informatics, Suez Canal University, Ismailia 41522, Egypt \\ ${ }^{3}$ Mathematics Department, Faculty of Science, King Abdulaziz University, P.O. Box 80203, Jeddah 21589, Saudi Arabia
}

Correspondence should be addressed to A. A. Elsadany; aelsadany1@yahoo.com

Received 15 May 2013; Revised 1 September 2013; Accepted 2 September 2013

Academic Editor: René Yamapi

Copyright (c) 2013 H. N. Agiza et al. This is an open access article distributed under the Creative Commons Attribution License, which permits unrestricted use, distribution, and reproduction in any medium, provided the original work is properly cited.

\begin{abstract}
This paper presents a new Cournot duopoly game. The main advantage of this game is that the outputs are nonnegative for all times. We investigate the complexity of the corresponding dynamical behaviors of the game such as stability and bifurcations. Computer simulations will be used to confirm our theoretical results. It is found that the chaotic behavior of the game has been stabilized on the Nash equilibrium point by using delay feedback control method.
\end{abstract}

\section{Introduction}

The classic model of oligopolies was proposed by the French mathematician A. Cournot [1]. He treated the case with nave expectations; at each time step players assume that the competitors produce the same quantity of goods already produced in the last period. The presence of complex dynamic phenomena in Cournot oligopoly models is well documented in the mathematical economics literature, starting from Rand [2] and Dana and Montrucchio [3]. The oligopoly market structure showing the action of only two companies is called duopoly. In duopoly game, each duopolist believes that he can calculate the quantity he should produce in order to maximize his profits. In fact, the properties of the best reply dynamics of Cournot duopoly games have been extensively studied by Puu $[4,5]$ who showed that trajectories may not converge to the Nash equilibrium and that complex trajectories are possible. Over the past decade, many researchers, such as Kopel [6], Bischi et al. [7], Ahmed and Agiza [8], and Agiza and Elsadany [9], have paid a great attention to the dynamics of games. The theoretical development of complex duopoly dynamics has been recently surveyed in $[10,11]$.

We consider a market consisting of a duopoly in which both firms, $F_{1}$ the domestic and $F_{2}$ the foreign firm, compete on quantities rather than price of production for a certain good. Let $q_{i}(t), i=1,2$, represent the quantity of $i$ th firm during the period $t=0,1,2, \ldots$ and $p_{i}$ the selling prices.
Suppose that the goods in a market are identical. The inverse demand functions of products come from the maximization by the representative consumer of the following fractional utility function:

$$
U\left(q_{1}, q_{2}\right)=\sqrt{q_{1}}+\sqrt{q_{2}}
$$

subject to the budget constraint

$$
p_{1} q_{1}+p_{2} q_{2}=1
$$

Using Lagrange multiplier to maximize utility function (1) subject to the budget constraint (2), one gets

$$
p_{1}=\frac{1}{\sqrt{q_{1}}\left(\sqrt{q_{1}}+\sqrt{q_{2}}\right)}, \quad p_{2}=\frac{1}{\sqrt{q_{2}}\left(\sqrt{q_{1}}+\sqrt{q_{2}}\right)} .
$$

In this paper, we present a new Cournot's duopoly game by using inverse demand function which was deduced in (3). The dynamical behavior of this game and the stability conditions for the Nash equilibrium are given. Theoretical analysis and numerical simulations of the system are made in detail. Finally, we give a feedback control to control chaos and stabilize the game.

The paper is organized as follows. In Section 2, we introduce the new model. In Section 3, we study the stability conditions of Nash equilibrium point. Section 4 is devoted to 
the analysis of the local bifurcations and the route to complex dynamics. Delay feedback control method is proposed to control chaos of the system in Section 5. Finally, a conclusion is drawn in Section 6.

\section{The Model}

Assume the inverse demand function as in (3). Further, the competitors have the cost functions which are

$$
C_{i}\left(q_{i}\right)=c_{i} \sqrt{q_{i}}, \quad i=1,2 .
$$

Then the profit of the two firm becomes

$$
\begin{aligned}
& \Pi_{1}\left(q_{1}, q_{2}\right)=\frac{\sqrt{q_{1}}}{\left(\sqrt{q_{1}}+\sqrt{q_{2}}\right)}-c_{1} \sqrt{q_{1}}, \\
& \Pi_{2}\left(q_{1}, q_{2}\right)=\frac{\sqrt{q_{2}}}{\left(\sqrt{q_{1}}+\sqrt{q_{2}}\right)}-c_{2} \sqrt{q_{2}} .
\end{aligned}
$$

The first firm maximizes $\Pi_{1}\left(q_{1}, q_{2}\right)$ with respect to $q_{1}$ and the second $\Pi_{2}\left(q_{1}, q_{2}\right)$ with respect to $q_{2}$. Equating the partial derivatives to zero, one can solve the reaction functions

$$
\begin{aligned}
& q_{1}=\phi_{1}\left(q_{2}\right)=\left(\sqrt[4]{\frac{q_{2}}{c_{1}^{2}}}-\sqrt{q_{2}}\right)^{2}, \\
& q_{2}=\phi_{2}\left(q_{1}\right)=\left(\sqrt[4]{\frac{q_{1}}{c_{2}^{2}}}-\sqrt{q_{1}}\right)^{2} .
\end{aligned}
$$

The long run map can now be formulated as

$$
\begin{aligned}
& q_{1}(t+1)=\left(\sqrt[4]{\frac{q_{2}}{c_{1}^{2}}}-\sqrt{q_{2}}\right)^{2}, \\
& q_{2}(t+1)=\left(\sqrt[4]{\frac{q_{1}}{c_{2}^{2}}}-\sqrt{q_{1}}\right)^{2} .
\end{aligned}
$$

The model has the advantage that $q_{1}(t), q_{2}(t)$ are nonnegative for all $t$. The dynamic system (7) has two fixed points: a trivial one $\left(q_{1}=0, q_{2}=0\right)$ and a nontrivial fixed, which is given by

$$
q_{1}^{*}=\frac{c_{2}^{2}}{\left(c_{1}+c_{2}\right)^{4}}, \quad q_{2}^{*}=\frac{c_{1}^{2}}{\left(c_{1}+c_{2}\right)^{4}} .
$$

This point, of course, is the intersection of the two reaction curves $\phi_{1}, \phi_{2}$. This point is called Nash equilibrium point. The profits of the duopolists at the Nash equilibrium can be calculated as

$$
\Pi_{1}=\frac{c_{2}^{2}}{\left(c_{1}+c_{2}\right)^{2}}, \quad \Pi_{2}=\frac{c_{1}^{2}}{\left(c_{1}+c_{2}\right)^{2}} .
$$

It is obvious that the firm with lower unit costs obtains the higher profit. The profits at the Nash equilibrium are the same as in Puu's game [4]. But the main advantage of this model than Puu's model is that outputs are nonnegative for all $t$.

\section{Nash Equilibrium and Local Stability}

We exclusively restrict attention to the analysis of the local stability properties of the positive Nash equilibrium $E$ of the two-dimensional system (7). For doing so, we build on the Jacobian matrix $J$ evaluated at any point $\left(q_{1}, q_{2}\right)$ as

$$
J\left(q_{1}, q_{2}\right)=\left[\begin{array}{cc}
0 & a_{12} \\
a_{21} & 0
\end{array}\right],
$$

where $a_{i j}=2\left(\sqrt[4]{q_{j} / c_{i}^{2}}-\sqrt{q_{j}}\right)\left(\left(1 / 4 c_{i}^{2}\right)\left(q_{j} / c_{i}^{2}\right)^{-3 / 4}-\left(q_{j}^{-1 / 2} / 2\right)\right)$, $i, j=1,2$. The Jacobian matrix evaluated at the Nash equilibrium point $E$ is

$$
J(E)=\left[\begin{array}{cc}
0 & \frac{c_{2}\left(c_{2}-c_{1}\right)}{2 c_{1}^{2}} \\
\frac{c_{1}\left(c_{1}-c_{2}\right)}{2 c_{2}^{2}} & 0
\end{array}\right] .
$$

For a system of two dimensions, the stability condition is that the eigenvalues remain within the unit circle. The characteristic equation of the Jacobian matrix (11) is

$$
\lambda^{2}-\frac{\left(c_{2}-c_{1}\right)\left(c_{1}-c_{2}\right)}{4 c_{1} c_{2}}=0 .
$$

The Nash equilibrium point loss stability occurs when

$$
\left|\lambda_{-} \lambda_{+}\right|=\left|\frac{\left(c_{2}-c_{1}\right)\left(c_{1}-c_{2}\right)}{4 c_{1} c_{2}}\right|=1 .
$$

Observe that the above condition can be solved for the ratio:

$$
k=\frac{c_{2}}{c_{1}}=3 \pm 2 \sqrt{2} .
$$

Then Nash equilibrium point is stable if and only if

$$
3-2 \sqrt{2}<k<3+2 \sqrt{2} .
$$

Condition (15) implies that, in the special case of $c_{1}=c_{2}$, the Nash equilibrium point is stable. When condition (15) is violated, the Nash equilibrium point is unstable, and increasing the ratio $c_{2} / c_{1}$, we can observe the period doubling sequence of bifurcations leading to chaos.

\section{Numerical Simulations and Fractal Dimension}

In this section, some numerical simulations are presented to confirm the above analytic results and to demonstrate complex dynamical behaviors of the system. Bifurcation diagrams, phase portraits, maximal Lyapunov exponents, and fractal dimensions are used to show complex dynamical behaviors.

Let $k=c_{2} / c_{1}$ be a varying parameter. Figure 1 exhibits the bifurcation diagram in $\left(k-q_{1}\right)$ plane. It is easy to see that map (7) changes from stable to unstable as $k$ increases from 5.7 to 9 and a bifurcation occurs at $k=5.725$. Also, Figure 2 exhibits the bifurcation diagram in $\left(k-q_{2}\right)$ plane. 


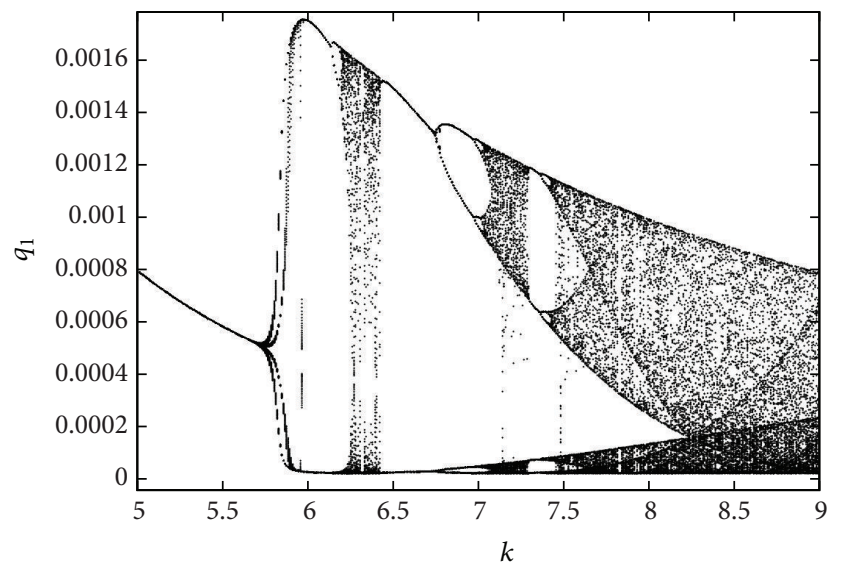

FIgURE 1: Bifurcation diagram of map (7) in $\left(k-q_{1}\right)$ plane.

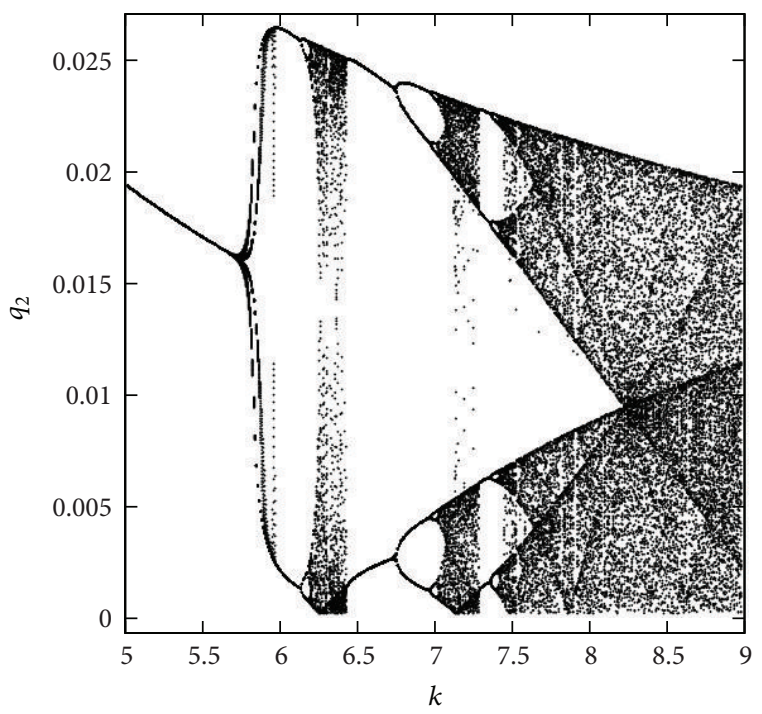

FIgURE 2: Bifurcation diagram of map (7) in $\left(k-q_{2}\right)$ plane.

In order to analyze the parameters influence in periodic and complex behavior, we compute the maximal Lyapunov exponents. The maximal Lyapunov exponent can be negative (stable fixed point), zero (bifurcation point), and positive (chaos). Moreover, comparing the bifurcation diagram and the values of Lyapunov exponent for the values of the parameters gave the same indications about stable and chaotic regions. In order to study the relations between the local stability of the Nash equilibrium point and the parameters $c_{1}$ and $c_{2}$ is based on maximal Lyapunov exponents. Figure 3 displays the related maximal Lyapunov exponents. A positive value of maximal Lyapunov exponents implies sensitive dependence on initial condition for chaotic behavior. From the maximal Lyapunov exponents diagram, it is easy to determine the parameter sets for which the system converges to cycles and chaotic behavior.

In the phase space, the chaotic motion is stochastic, and its trajectory is never closed in a given region. An attractor is informally described as strange if it has a noninteger

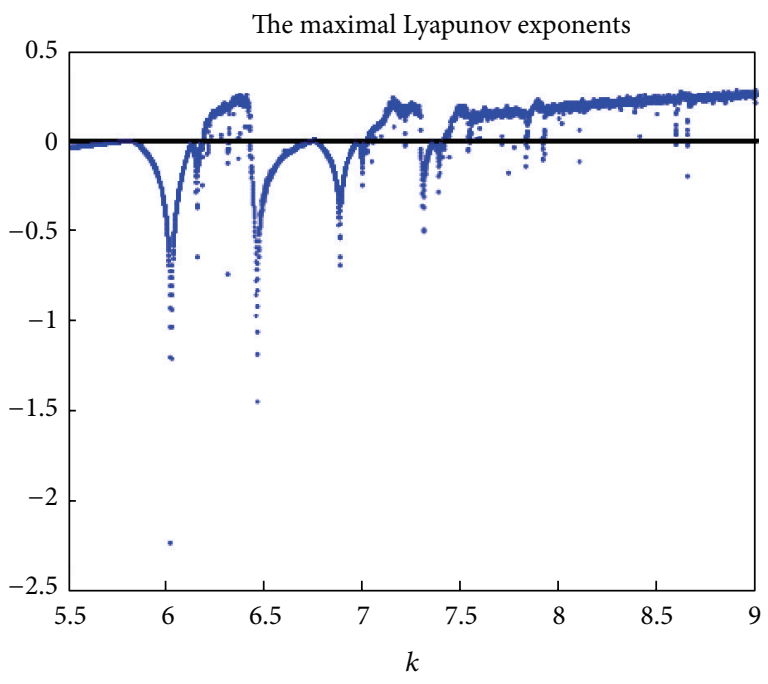

Figure 3: The maximal Lyapunov exponent with respect to $k$.

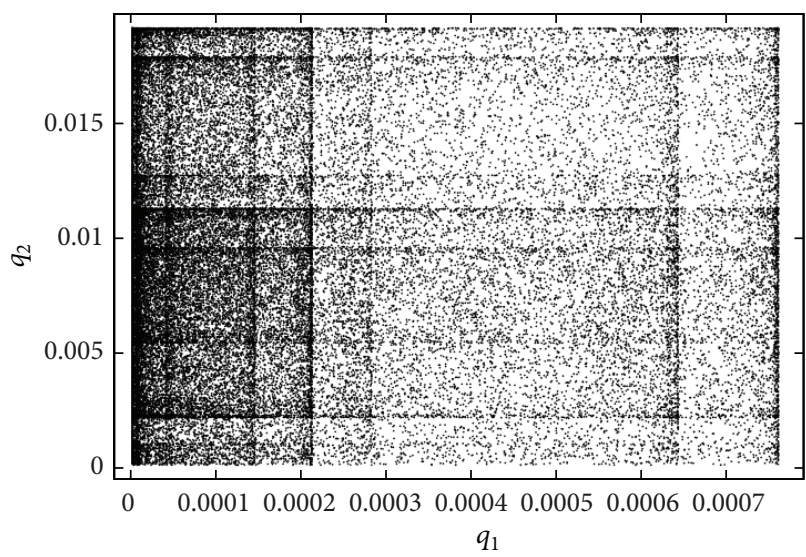

FIGURE 4: Chaotic attractor of map (7) at $k=9$.

dimension. The obvious character of the chaotic attractor is the exponential separation of two adjacent trajectories, which shows the sensitive dependence on the initial conditions of the chaotic system. The chaotic attractor of system map (7) at $k=9$ is shown in Figure 4 .

As known, the sensitivity to initial conditions is a characteristic of a deterministic chaos. In order to show the sensitivity to initial conditions of system map (7), we have computed two orbits of the variable $q_{1}$ whose coordinates of initial conditions differ by 0.00001 . Figure 5 depicts the orbits of $q_{1}$ with initial $\left(q_{1,0}, q_{2,0}\right)=(0.3,0.2)$ and $\left(q_{1,0}, q_{2,0}\right)=$ $(0.30001,0.2)$ at $k=9$. The orbits rapidly separate each other, thus suggesting the existence of deterministic chaotic.

Chaotic attractors are typically characterized by fractal dimensions. We examine the important characteristic of neighboring chaotic orbits to see how rapidly they separate each other. The Lyapunov dimension (Kaplan and Yorke conjecture [12]) is defined as follows:

$$
d_{L}=j+\frac{\sum_{i=1}^{j} \Lambda_{i}}{\left|\Lambda_{j}\right|}
$$




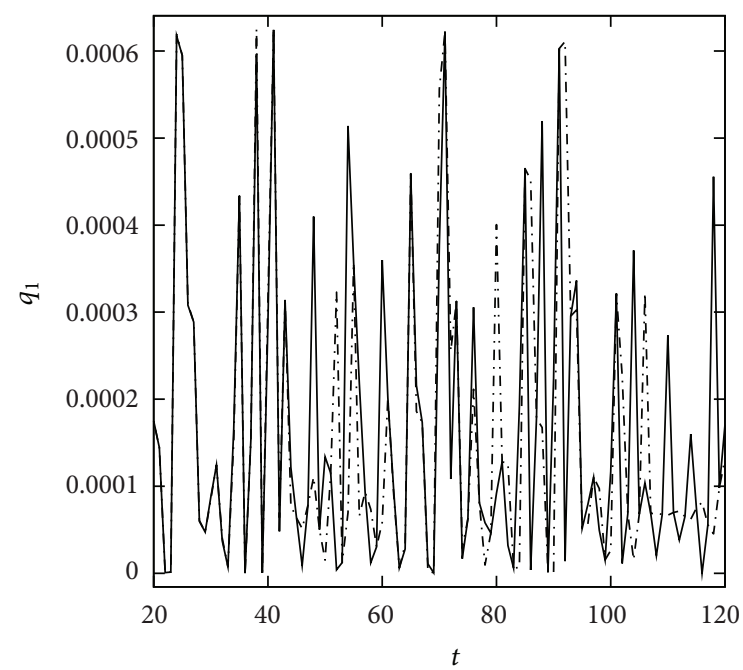

FIGURE 5: Sensitivity dependence on initial conditions $\left(q_{1}\right.$ versus time).

with $\Lambda_{1}, \Lambda_{2}, \ldots, \Lambda_{n}$, where $j$ is the largest integer such that $\sum_{i=1}^{i=j} \Lambda_{i} \geq 0$ and $\sum_{i=1}^{i=j+1} \Lambda_{i}<0$.

In our work, the two-dimensional map (7) has a Lyapunov dimension

$$
d_{L}=1+\frac{\Lambda_{1}}{\left|\Lambda_{2}\right|}, \quad \Lambda_{1}>0>\Lambda_{2} .
$$

By the definition of Lyapunov dimension and simulation of the computer, we have the Lyapunov dimension of the strange attractor of system (7). At the parameter value $k=9$, system (7) has two different Lyapunov exponents, $\Lambda_{1}=0.37$ and $\Lambda_{2}=-1.26$. Therefore, system (7) has a fractal dimension $d_{L}=1+(0.37 / 1.26) \approx 1.3$. Then system $(7)$ exhibits a fractal structure, and its attractor has the fractal dimension $d_{L} \approx 1.3$.

\section{Controlling Chaos}

Over the past two decades, there has been tremendous interest in controlling bifurcation and chaos in dynamical systems [13]. Although a lot of methods have been proposed, many of them cannot be directly applied to the control of oscillations and chaos in economic systems due to the complexity of the economics and the limitation of the allowable control in applications. In order to control chaotic behavior of economic system (7), we apply Pyragas' method [14]. In Pyragas' method, control input is based on the difference between the $T$-time delayed state and the current state, where $T$ denotes a period of the stabilized orbits. So the controlled system is given by

$$
q(t+1)=f(q(t), u(t)),
$$

where $u(t)$ is the input signal, $q(t)$ is the state variable, and $f$ is a nonlinear vector field. Pyragas proposed the following feedback in order to stabilize a $T$-periodic orbit:

$$
u(t)=w(q(t+1-T)-q(t+1)) ; \quad t>T,
$$

where $T$ is the time delay and $w$ is the controlling parameter.

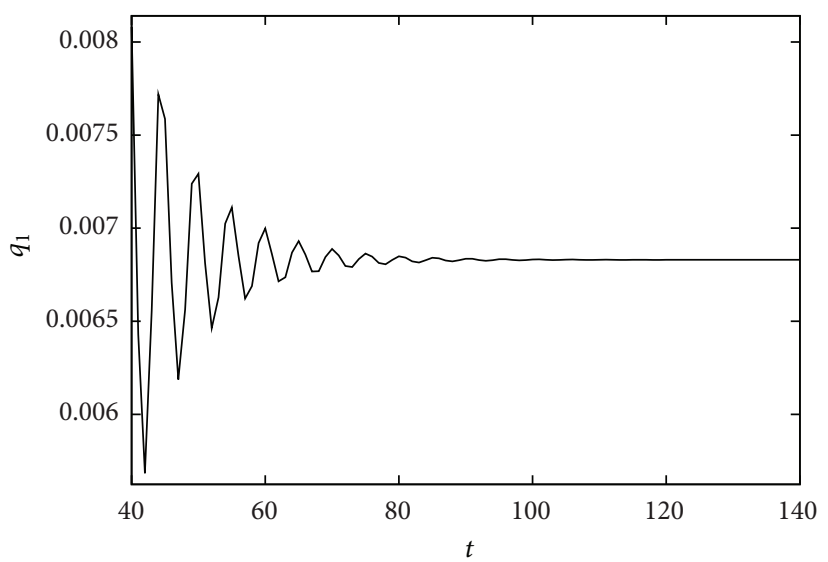

FIgURE 6: Control of chaos in the model using delayed feedback control method.

In $[15,16]$, how the delay feedback control method can be applied to control chaos in two economic models has been presented. The adjustment method based on the marginal profit is one reasonable way for the player to adjust his quantity decision, but it is not the only way. A producer can use a feedback of his decision-making variable to control the adjustment magnitude. Elabbasy et al. [16] have considered such a feedback control in their triopoly Cournot model. Chaos in Cournot game means that if one firm changes its output even slightly, then, on the long run, large unpredictable changes will occur in the outputs of all firms. This is not a favorable situation. Therefore, all firms should try to control chaos. In this section, we show that one firm can control chaos (the player himself). We apply this technique to control chaotic behavior for the dynamic game (7). We set $T=1$; then the controlled system can be expressed as follows:

$$
\begin{gathered}
q_{1}(t+1)=\frac{1}{1+w}\left(w q_{1}+\left(\sqrt[4]{\frac{q_{2}}{c_{1}^{2}}}-\sqrt{q_{2}}\right)^{2}\right) \\
q_{2}(t+1)=\left(\sqrt[4]{\frac{q_{1}}{c_{2}^{2}}}-\sqrt{q_{1}}\right)^{2} .
\end{gathered}
$$

Then the Jacobian matrix at Nash equilibrium point is

$$
J\left(q_{1}^{*}, q_{2}^{*}\right)=\left[\begin{array}{cc}
\frac{w}{1+w} & \frac{k(k-1)}{2(1+w)} \\
\frac{1-k}{2 k^{2}} & 0
\end{array}\right]
$$

By using the values of parameters $\left(c_{1}=1\right.$ and $\left.c_{2}=9\right)$ where chaos exists in system (7), Jacobian matrix (21) has the form

$$
J=\left[\begin{array}{cc}
\frac{w}{1+w} & \frac{36}{(1+w)} \\
\frac{-4}{81} & 0
\end{array}\right]
$$


According to the Jury conditions [17], only when $w>25 / 18$, all the eigenvalues of the system are less than 1 . This means that also the system is stable around the Nash equilibrium point.

Numerical simulations are performed to show the effectiveness of the proposed control scheme. We have performed numerical simulations to see how the state delayed feedback method controls the unstable Nash equilibrium. Parameter values are fixed as $c_{1}=1$ and $c_{2}=9$, the initial condition $q_{1}(0)=0.3$ and $q_{2}(0)=0.2$, and the control parameter $w=1.5$. Figure 6 shows that a chaotic trajectory is stabilized to the Nash equilibrium. From Figure 6, one can see that the system is controlled from a chaotic state to a stable state when $w=1.5$.

\section{Conclusion}

In the paper, we developed a simple model of Cournot duopoly game. This model is a modification to Puu Cournot duopoly game [4] where the values of the output quantities $q_{1}$ and $q_{2}$ can take positive values as in real life markets. The local stability and bifurcations of fixed points were investigated. From bifurcation diagrams, the basic properties of the game are presented. The numerical simulation shows the complex dynamical behaviors of the duopoly game. Stabilization of the chaotic behavior can be achieved by applying delay feedback control method.

\section{Acknowledgments}

The authors thank the anonymous reviewers for their helpful comments and suggestions. They thank Professor E. Ahmed for his valuable comments.

\section{References}

[1] A. Cournot, Recherches sur les Prinicipes Mathematiques de la Theorie des Richesses, Hachette, Paris, France, 1838.

[2] D. Rand, "Exotic phenomena in games and duopoly models," Journal of Mathematical Economics, vol. 5, no. 2, pp. 173-184, 1978.

[3] R.-A. Dana and L. Montrucchio, "Dynamic complexity in duopoly games," Journal of Economic Theory, vol. 40, no. 1, pp. 40-56, 1986.

[4] T. Puu, "Chaos in duopoly pricing," Chaos, Solitons and Fractals, vol. 1, no. 6, pp. 573-581, 1991.

[5] T. Puu, "Complex dynamics with three oligopolists," Chaos, Solitons and Fractals, vol. 7, no. 12, pp. 2075-2081, 1996.

[6] M. Kopel, "Simple and complex adjustment dynamics in Cournot duopoly models," Chaos, Solitons and Fractals, vol. 7, no. 12, pp. 2031-2048, 1996.

[7] G.-I. Bischi, M. Gallegati, and A. Naimzada, "Symmetrybreaking bifurcations and representative firm in dynamic duopoly games," Annals of Operations Research, vol. 89, pp. 253272,1999 .

[8] E. Ahmed and H. N. Agiza, "Dynamics of a cournot game with n-competitors," Chaos, Solitons and Fractals, vol. 9, no. 9, pp. 1513-1517, 1998.
[9] H. N. Agiza and A. A. Elsadany, "Nonlinear dynamics in the Cournot duopoly game with heterogeneous players," Physica A, vol. 320, pp. 512-524, 2003.

[10] T. Puu, Oligopoly: Old Ends-New Means, Springr, 2011.

[11] L. Chen and G. Chen, "Controlling chaos in an economic model," Physica A, vol. 374, no. 1, pp. 349-358, 2007.

[12] J. L. Kaplan and J. A. Yorke, "Preturbulence: a regime observed in a fluid flow model of Lorenz," Communications in Mathematical Physics, vol. 67, no. 2, pp. 93-108, 1979.

[13] G. Chen and X. Dong, From Chaos to Order: Perspectives, Methodologies, and Applications, World Scientific, Singapore, 1998.

[14] K. Pyragas, "Continuous control of chaos by self-controlling feedback," Physics Letters A, vol. 170, no. 6, pp. 421-428, 1992.

[15] J. A. Holyst and K. Urbanowicz, "Chaos control in economical model by time-delayed feedback method," Physica A, vol. 287, no. 3-4, pp. 587-598, 2000.

[16] E. M. Elabbasy, H. N. Agiza, and A. A. Elsadany, "Analysis of nonlinear triopoly game with heterogeneous players," Computers and Mathematics with Applications, vol. 57, no. 3, pp. 488499, 2009.

[17] E. I. Jury and J. Blanchard, "A stability test for linear discrete systems in table form," Proceedings of the Institute of Radio Engineers, vol. 49, pp. 1947-1948, 1961. 


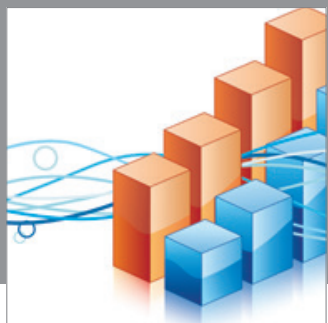

Advances in

Operations Research

mansans

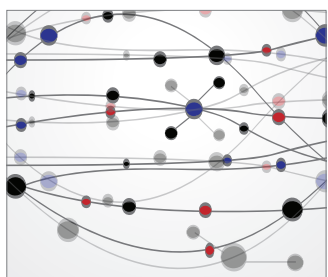

The Scientific World Journal
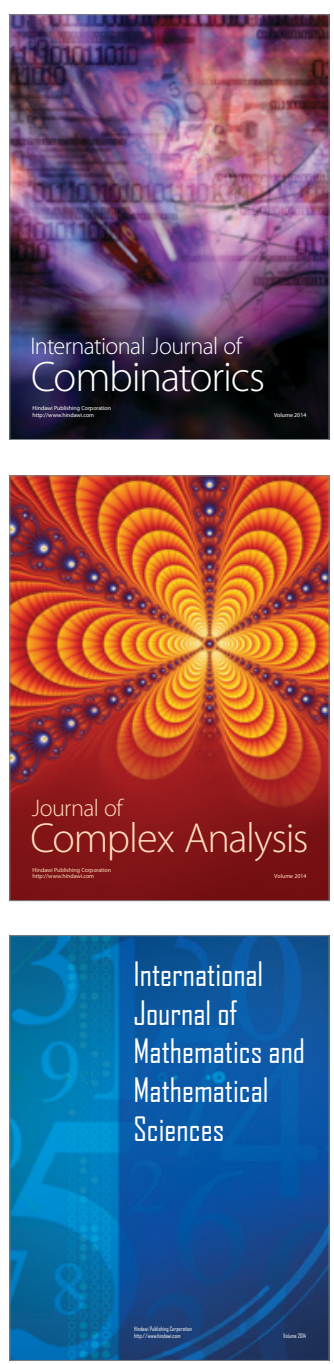
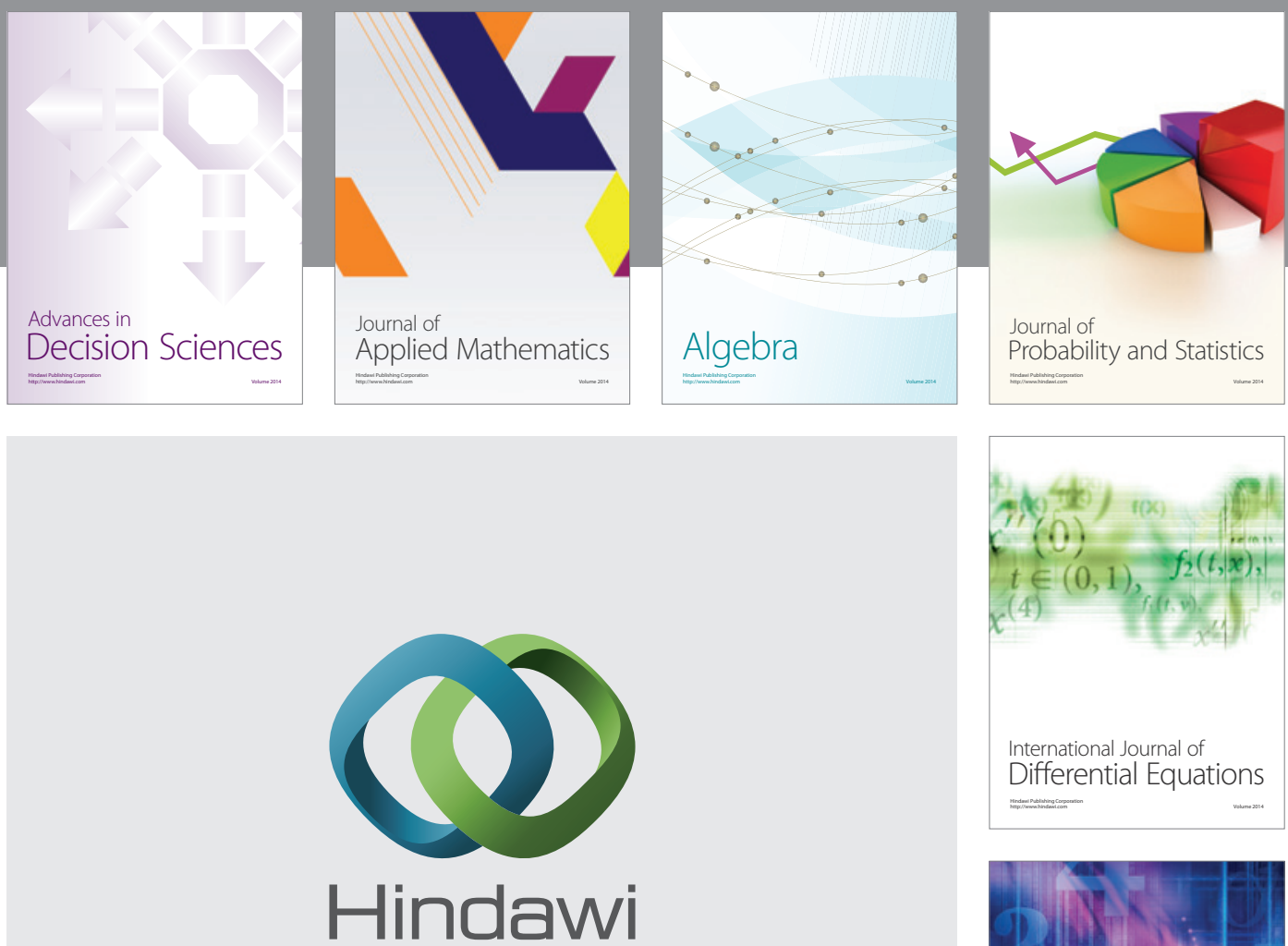

Submit your manuscripts at http://www.hindawi.com
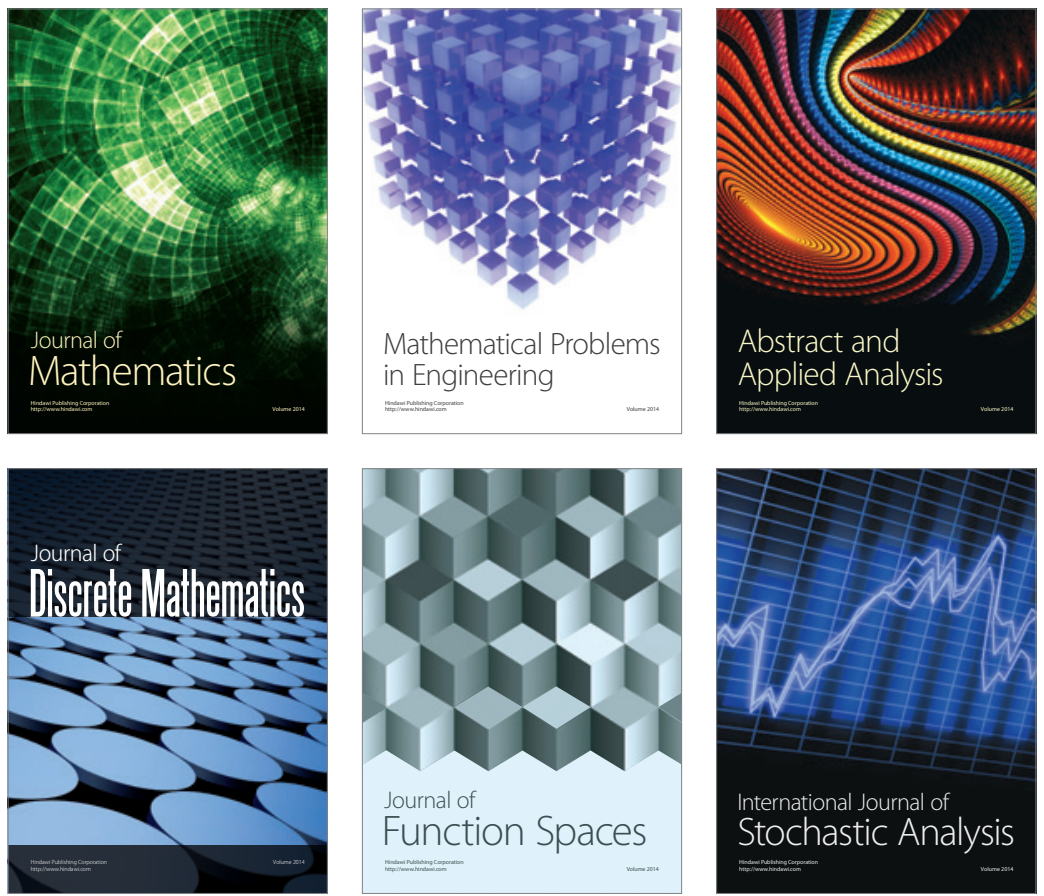

Journal of

Function Spaces

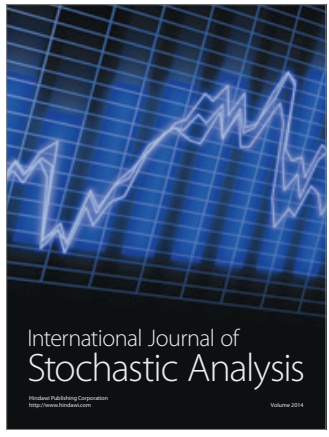

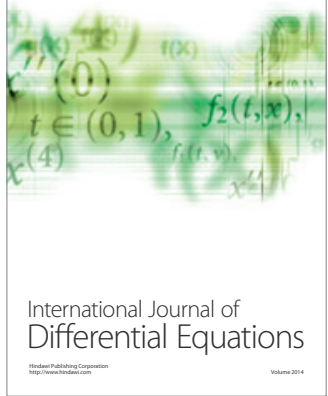
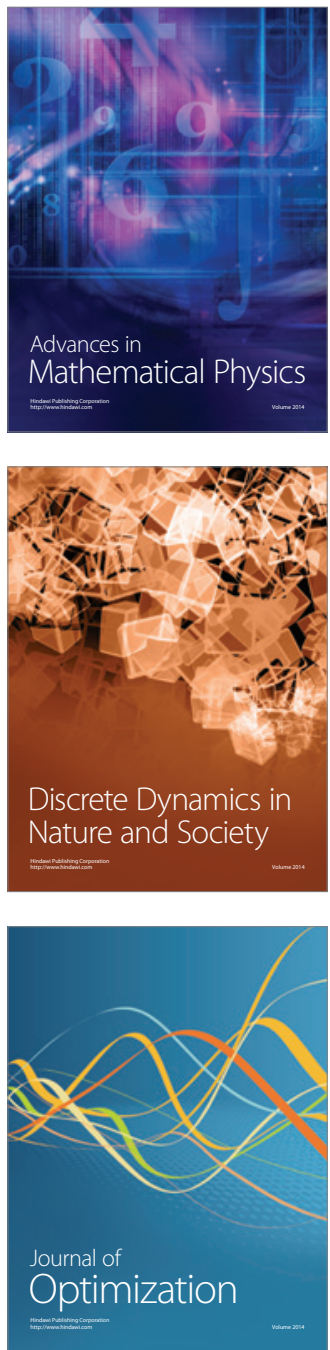